\title{
Global Protease Activity Profiling Identifies HER2-driven Proteolysis in Breast Cancer
}

Eugenia C. Salcedo ${ }^{1}$, Michael B. Winter ${ }^{2, a}$, Natalia Khuri ${ }^{3, b}$, Giselle M. Knudsen ${ }^{2, c}$, Andrej Sali, Charles S. Craik ${ }^{2 *}$

\section{Author affiliation:}

${ }^{1}$ Chemistry and Chemical Biology Graduate Program, ${ }^{2}$ Department of Pharmaceutical Chemistry,

${ }^{3}$ Department of Bioengineering and Therapeutic Sciences, University of California- San Francisco, San Francisco, CA 94158

aPresent address: CytomX Therapeutics, Inc. South San Francisco, CA 94080

bPresent address: Department of Computer Science, Wake Forest University, Winston-Salem, NC 27109

'Present address: Alaunus Biosciences, Inc. San Francisco, CA 94107

${ }^{*}$ Corresponding author:

Charles S. Craik

University of California, San Francisco

Genentech Hall, Room S-514

$60016^{\text {th }}$ Street, Box 2280

San Francisco, CA 94158

Phone: (415) 4768146

Email: charles.craik@ucsf.edu 


\section{SUPPORTING INFORMATION}

\section{Supplementary Figures:}

Figure S1. Analysis of differentially expressed proteases and protease inhibitors in breast cancer subtypes.

Figure S2. Proteomic analysis in conditioned media of cathepsin lysosomal cysteine proteases in breast cancer cell lines.

Figure S3. Global protease substrate specificity profiles for panel of breast cancer cell lines of distinct molecular subtypes.

Figure S4. Evaluation of cellular proliferation in MCF10A control and HER2-expressing cells.

Figure S5. Global protease substrate specificity profiles for MCF10A control and MCF10A HER2expressing cell lines.

Figure S6. Evaluation of inhibitors treatment on cellular proliferation in MCF10A control and MCF10A HER2-expressing cells.

\section{Supplementary Tables:}

Supplementary tables are provided as excel files.

Table S1. Proteomic analysis of extracellular proteins in 9 breast cancer cell lines.

Table S2. Peptide cleavage products (P4-P4') for panel of breast cancer cell lines by MSP-MS.

Table S3. Proteomic analysis of extracellular proteins in an isogenic HER2 cell line model.

Table S4. Peptide cleavage products (P4-P4') for MCF10A control and MCF10A HER2 by MSP-MS. 
A

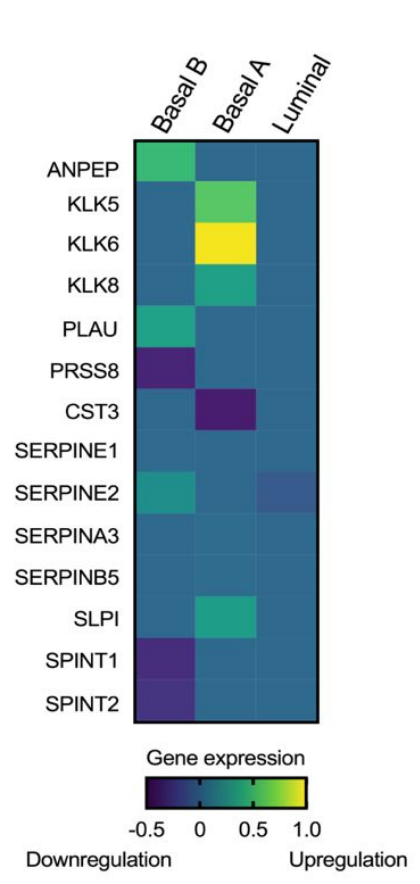

B

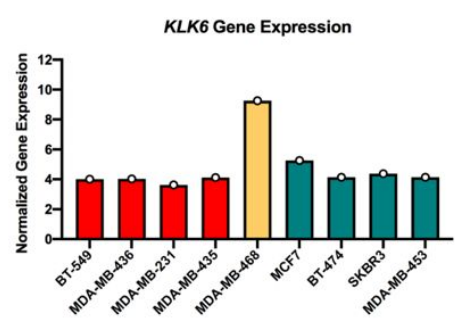

PLAU Gene Expression

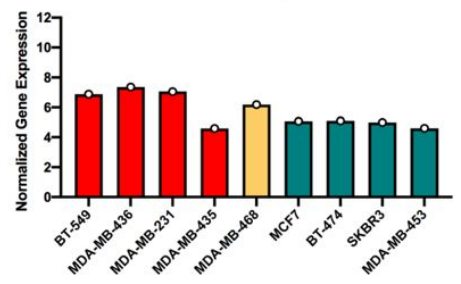

ANPEP Gene Expression

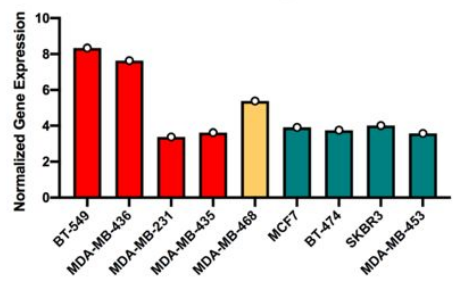

SLPIGene Expression

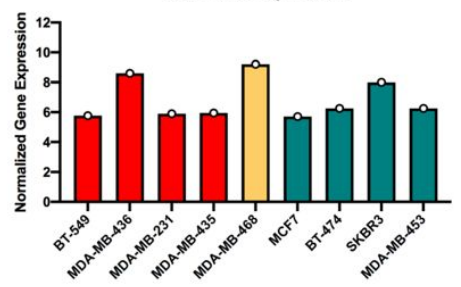

CST3 Gene Expression

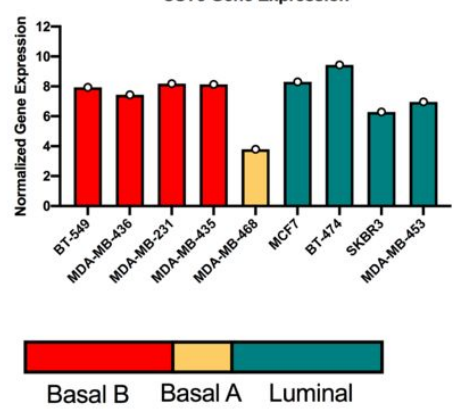

KLK6 Protein Expression

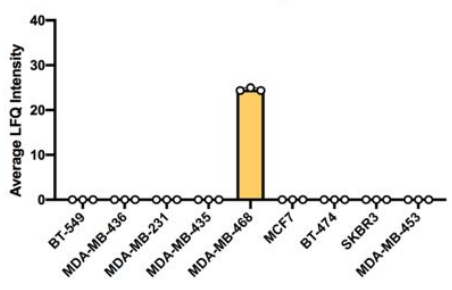

PLAU Protein Expression

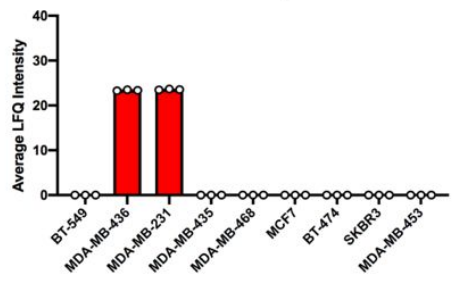

ANPEP Protein Expression

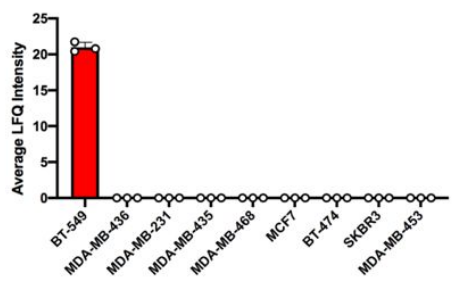

SLPI Protein Expression

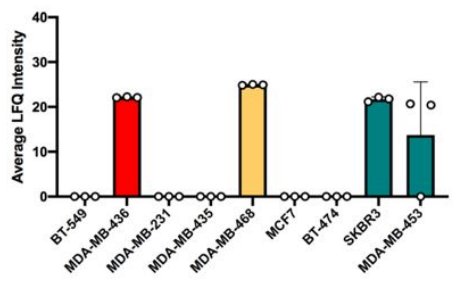

CST3 Protein Expression

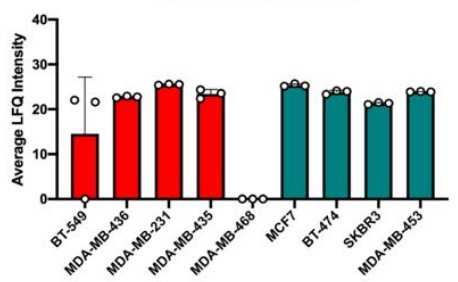

Figure S1. Analysis of differentially expressed proteases and protease inhibitors in breast cancer subtypes. (A) Proteases and protease inhibitors identified by transcriptional analysis to be differentially associated with distinct molecular subtypes of breast cancer (9). (B) Proteomic 
analysis on conditioned media of selected protease and protease inhibitors previously identified to be differentially expressed at the transcriptional level (9). 
A

CTSL Gene Expression

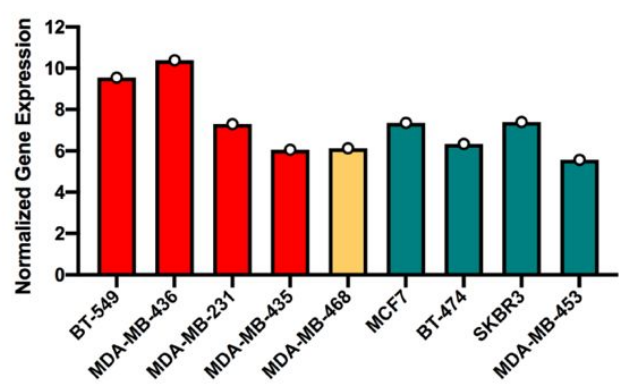

CTSB Gene Expression

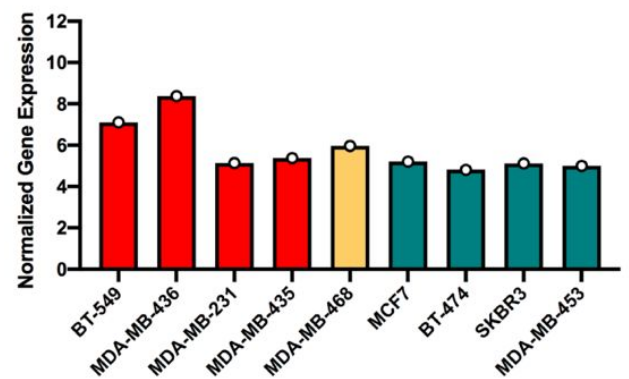

CTSZ Gene Expression

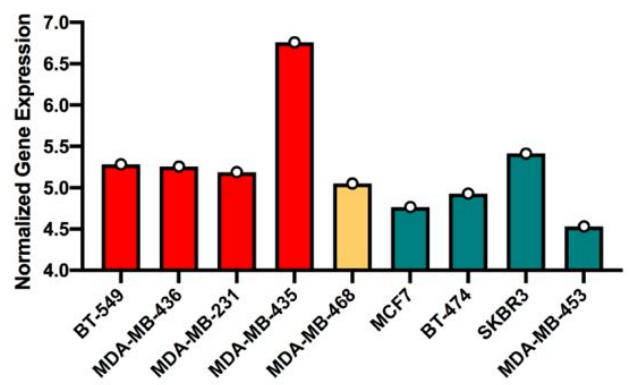

CTSD Gene Expression

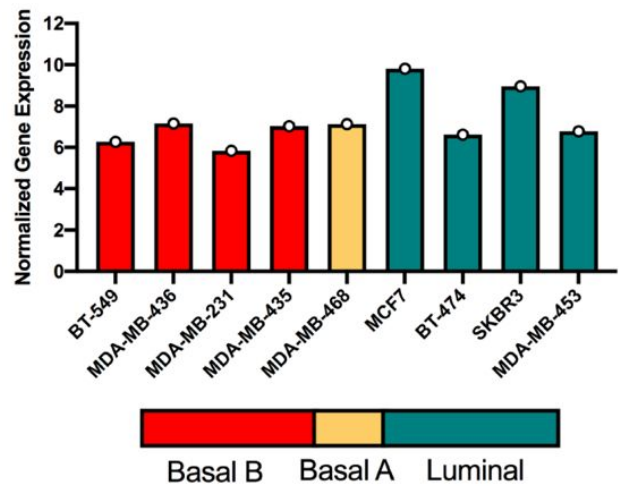

CTSL Protein Expression

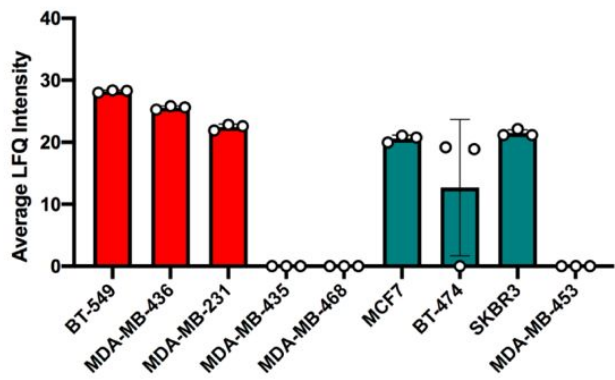

CTSB Protein Expression

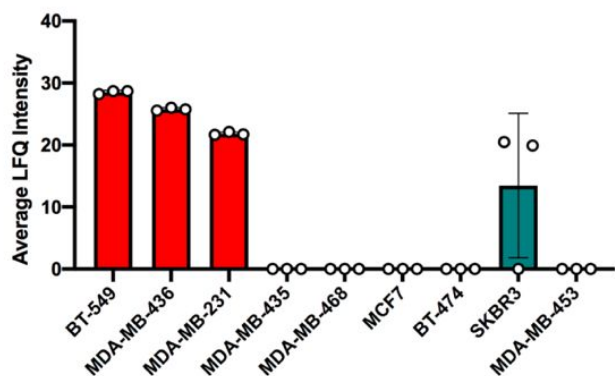

CTSZ Protein Expression

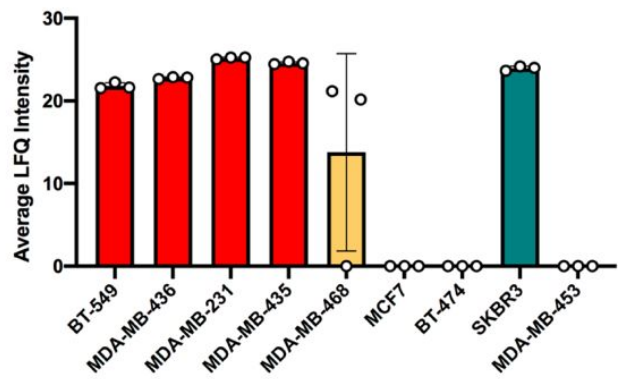

CTSD Protein Expression

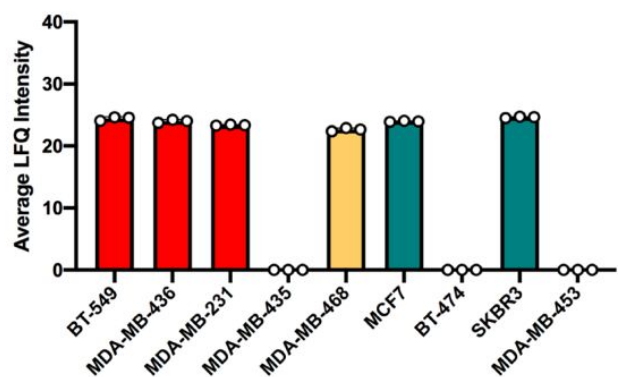

Figure S2. Proteomic analysis on conditioned media of cathepsin lysosomal cysteine proteases found to be differentially expressed by transcriptional analysis in breast cancer cell lines (9). 

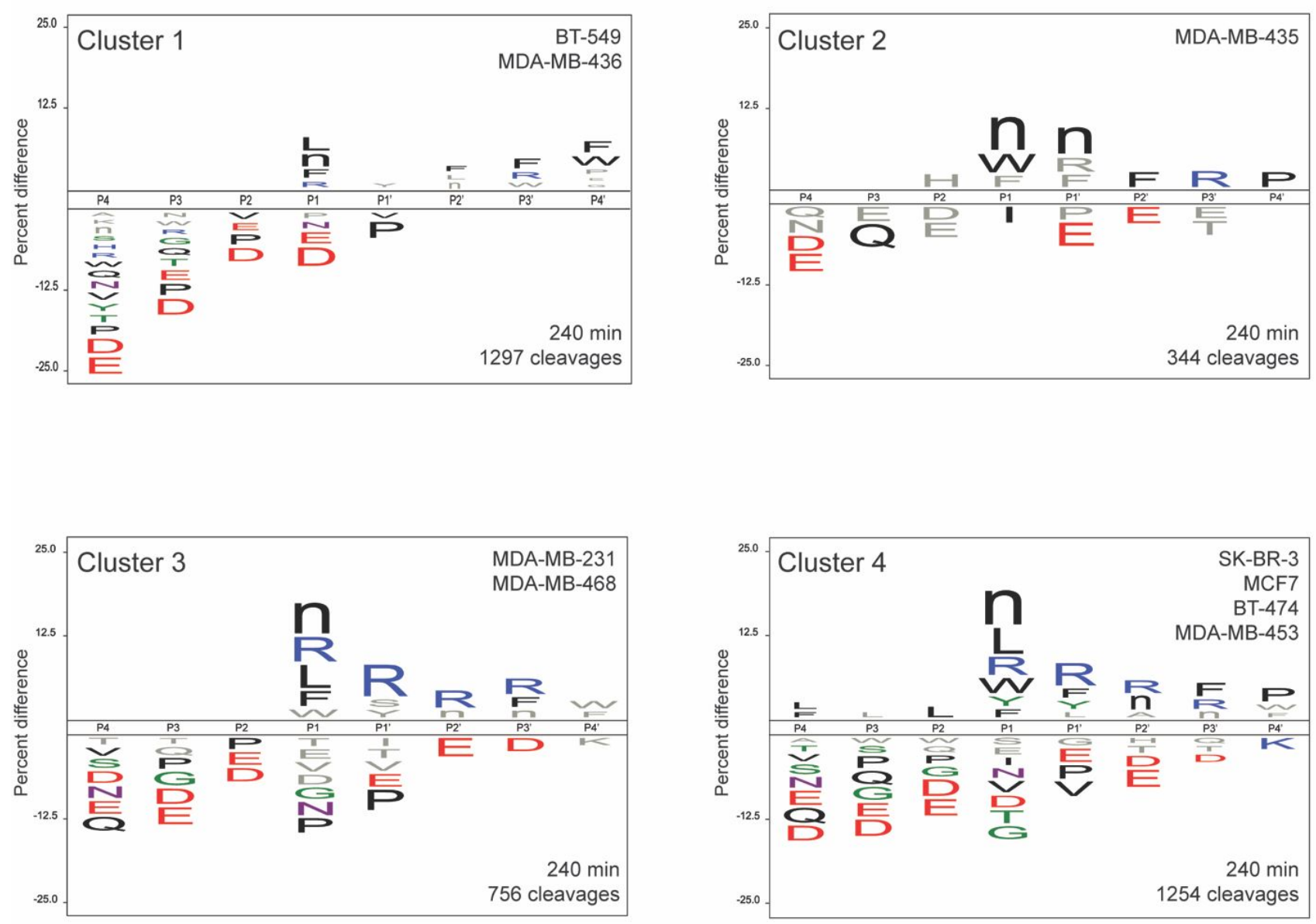

Figure S3. Global protease substrate specificity profiles for panel of breast cancer cell lines of distinct molecular subtypes (Table 1). iceLogo frequency plot (22) was generated based on cleavages used for clustering analysis (Figure 2) ( $n=1297$ for cluster 1, $n=344$ for cluster 2, $n=$ 756 for cluster $3, n=1254$ for cluster 4$)$. 
A

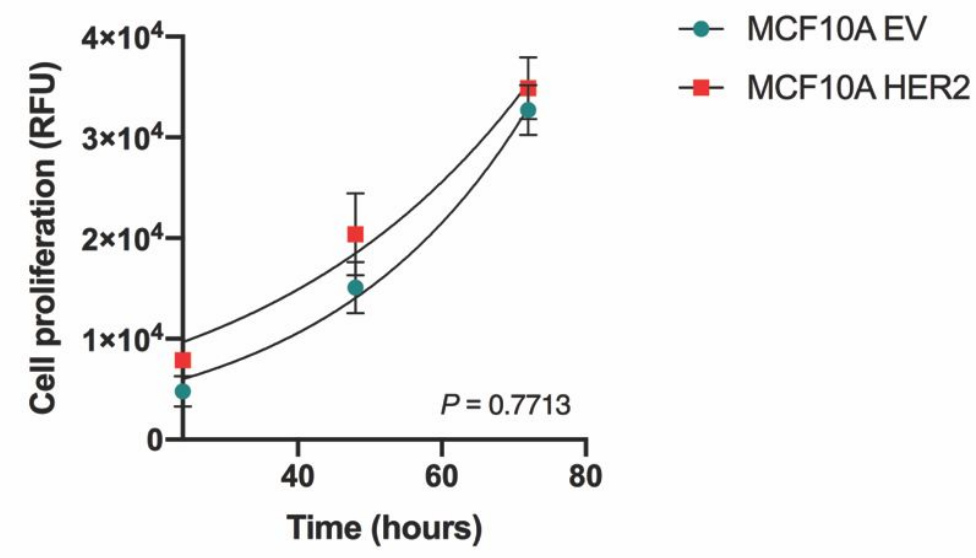

Figure S4. Evaluation of cellular proliferation in MCF10A control and HER2-expressing cells. HER2 expression in MCF10A cells did not result in significant changes on cellular proliferation. Results are given as mean relative fluorescence units (RFU) \pm SD of 4 wells. 

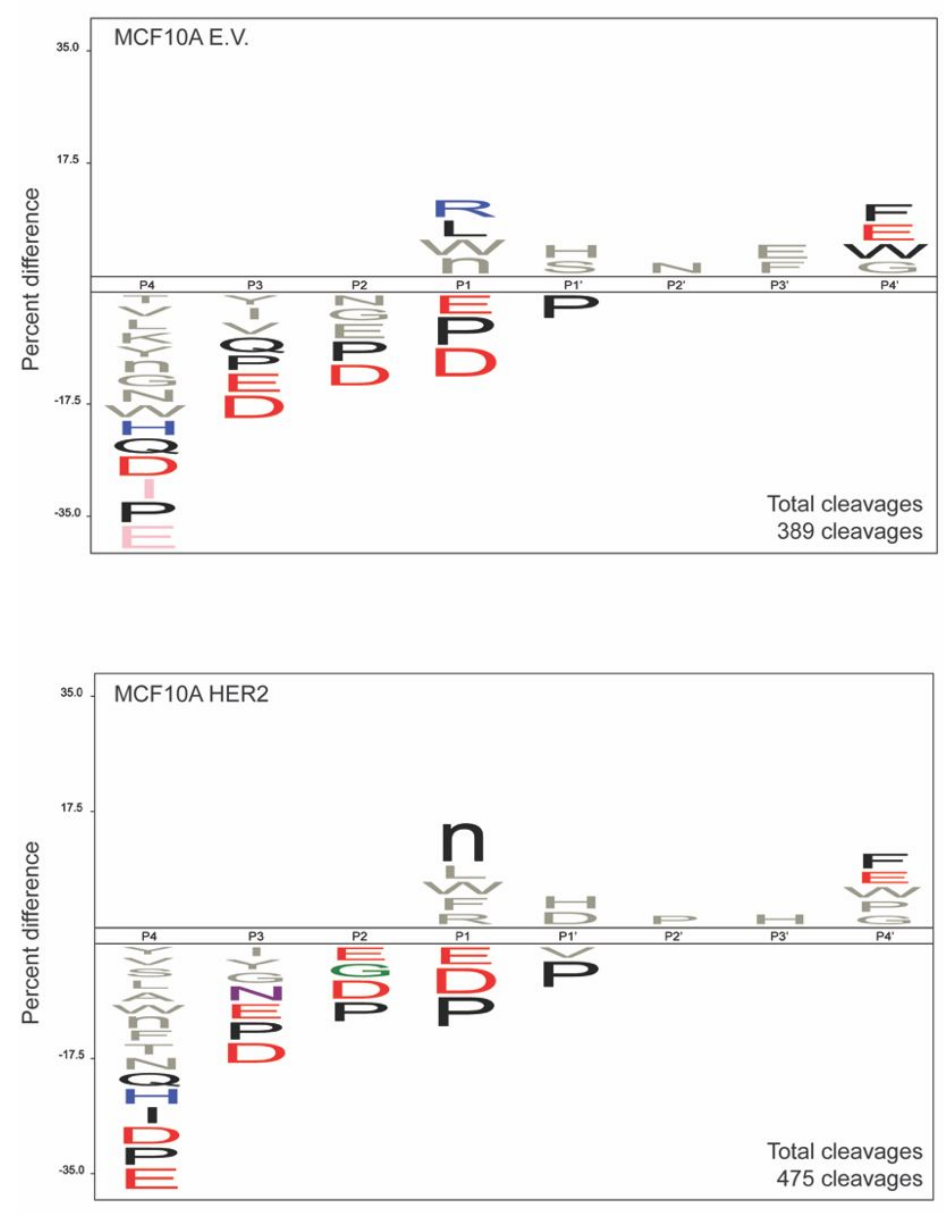

Figure S5. Global protease substrate specificity profiles for MCF10A control and MCF10A HER2expressing cell lines. iceLogo frequency plot (22) of total cleavages observed throughout the assay time course and present in at least two replicates for MCF10A control $(n=389)$ and MCF10A HER2-expressing $(n=475)$ cells. 
A

Cl-1033 (HER2 Inhibitor)

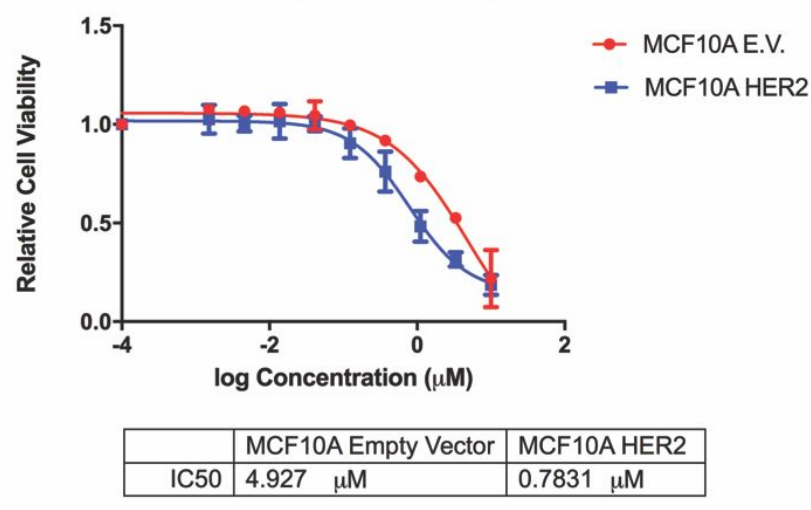

B

UK-383367 (BMP-1 inhibitor)

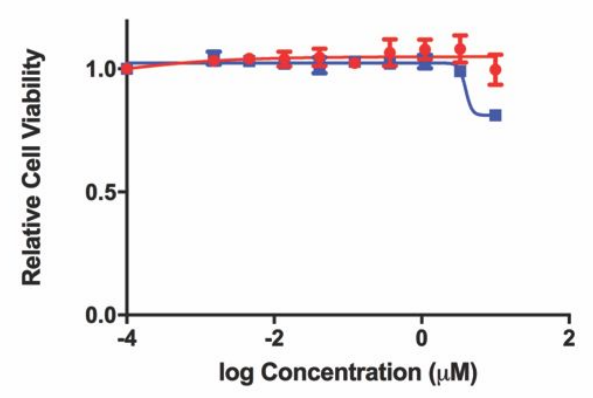

C

Galunisertib (TGF- $\beta$ Receptor Inhibitor)

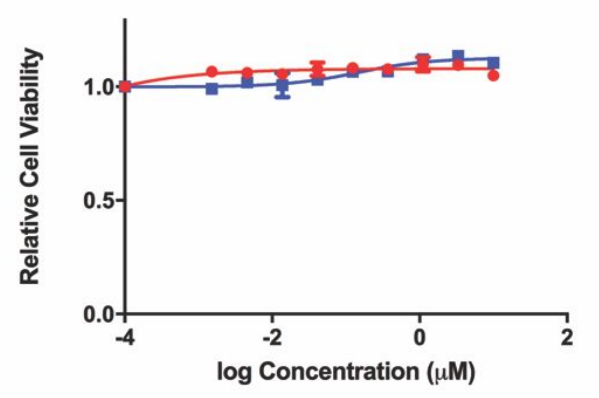

Figure S6. Evaluation of inhibitors treatment on cellular proliferation in MCF10A control and MCF10A HER2-expressing cells. Cell lines were treated with HER2, BMP-1 and TGF- $\beta$ receptor inhibitor for 72 hours, and relative cell proliferation was measured using Cell Titer-Glo. IC $\mathrm{C}_{50}$ values were calculated where appropriate. 